\title{
Extension of the Lorenz-Mie-Debye method for electromagnetic scattering to the time-domain
}

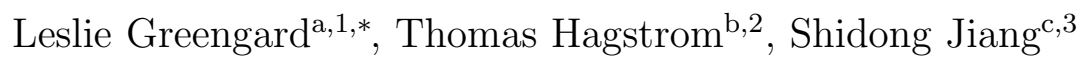 \\ ${ }^{a}$ Courant Institute of Mathematical Sciences, New York University, New York, NY \\ 10012. \\ ${ }^{b}$ Department of Mathematics, Southern Methodist University, PO Box 750156, Dallas, \\ $T X 75275$. \\ ${ }^{c}$ Department of Mathematical Sciences, New Jersey Institute of Technology, Newark, \\ New Jersey 07102.
}

\begin{abstract}
In this paper, we extend the frequency domain Lorenz-Mie-Debye formalism for the Maxwell equations to the time domain. In particular, we show that the problem of scattering from a perfectly conducting sphere can be reduced to the solution of two scalar wave equations - one with Dirichlet boundary conditions and the other with Robin boundary conditions. An explicit, stable, and high-order numerical scheme is then developed, based on our earlier treatment of the scalar case. This new representation may provide some insight into transient electromagnetic phenomena, and can also serve as a reference solution for general purpose time-domain software packages.
\end{abstract}

Keywords: Maxwell equations, Debye potentials, Mie series, vector spherical harmonics.

2000 MSC: 65M70, 78A40, 78M16

\footnotetext{
*Corresponding author.

Email addresses: greengard@courant.nyu.edu (Leslie Greengard), thagstrom@smu .edu (Thomas Hagstrom), shidong.jiang@njit.edu (Shidong Jiang)

${ }^{1}$ Supported in part by the Office of the Assistant Secretary of Defense for Research and Engineering and AFOSR under NSSEF Program Award FA9550-10-1-0180 and in part by the Department of Energy under contract DEFG0288ER25053.

${ }^{2}$ Supported in part by the Army Research Office under agreement W911NF-09-1-0344 and by the NSF under grant DMS-1418871.

${ }^{3}$ Supported in part by NSF under grant DMS-1418918.
} 


\section{Introduction}

The Lorenz-Mie-Debye solution to the Maxwell equations $[4,11,13]$ is a standard tool for their analysis in the frequency domain, when an incoming field impinges on a spherical scatterer. Not only has substantial intuition about wave scattering been gleaned in this manner, the corresponding exact solution serves as a standard benchmark for investigating the accuracy and convergence behavior of computational electromagnetics software packages. It is interesting to note that the Lorenz-Mie-Debye solution (often referred to as the "Mie series") is also used in benchmarking time-domain codes, essentially because an easily computable exact solution in the time domain is not available in the mathematical physics literature. As a result, one typically carries out a long simulation in time, followed by Fourier transformation and a mode by mode comparison of accuracy at each frequency.

Here, we derive a natural extension of the Lorenz-Mie-Debye formalism to the time domain, leading to an arbitrarily high order accurate solver. More precisely, we show that the problem of scattering from a perfect conductor can be reduced to the solution of two scalar wave equations - one with Dirichlet boundary conditions and one with Robin boundary conditions. These scalar problems have been studied in detail in [6], where a high-order, semi-analytic approach was derived, avoiding marching in space-time and free from numerical dispersion error.

After describing the underlying theory, we demonstrate the performance of the method on electrically large scattering problems. Letting $N_{S}^{2}$ denote the number of discretization points on the unit sphere (the scatterer) and $N_{T}$ the number of discretization points in the time interval $[0, T-R+1]$, the computational cost for evaluating the solution on a sphere of radius $R$ at time $T$ is $O\left(N_{T} N_{S}^{3}\right)$. For very large-scale problems, fast spherical harmonic transforms could be used to reduce the cost to $O\left(N_{T} N_{S}^{2} \log N_{S}\right)$ work, but with a larger constant (see, for example, $[1,18]$ and the references therein).

\section{A time-domain version of the Lorenz-Mie-Debye formalism}

Let us turn now the problem of electromagnetic scattering in vacuum, governed by the time-dependent Maxwell equations in non-dimensionalized 
units (for which the speed of light $c=1$ ):

$$
\begin{aligned}
\nabla \times \mathbf{E}^{\mathrm{tot}} & =-\frac{\partial \mathbf{B}^{\mathrm{tot}}}{\partial t}, & \nabla \times \mathbf{B}^{\mathrm{tot}} & =\frac{\partial \mathbf{E}^{\mathrm{tot}}}{\partial t}, \\
\nabla \cdot \mathbf{E}^{\mathrm{tot}} & =0, & \nabla \cdot \mathbf{B}^{\mathrm{tot}} & =0,
\end{aligned}
$$

where $\left\{\mathbf{E}^{\text {tot }}, \mathbf{B}^{\text {tot }}\right\}$ are the total electric and magnetic fields. Using the language of scattering theory, we let $\mathbf{E}^{\text {tot }}=\mathbf{E}+\mathbf{E}^{\text {inc }}$ and $\mathbf{B}^{\text {tot }}=\mathbf{B}+\mathbf{B}^{\text {inc }}$, where $\left\{\mathbf{E}^{\text {inc }}, \mathbf{B}^{\text {inc }}\right\}$ are known incident fields, and $\{\mathbf{E}, \mathbf{B}\}$ are the unknown scattered fields of interest.

We will assume throughout the paper that we have zero initial conditions for the scattered field; that is the incident field does not interact with the scatterer until $t>0$. In the case of a perfect conductor, boundary conditions for $\mathbf{E}$ and $\mathbf{B}$ are well-known to take the form [7]:

$$
\begin{aligned}
\mathbf{n} \times \mathbf{E} & =-\mathbf{n} \times \mathbf{E}^{\text {inc }}, \\
\mathbf{n} \cdot \mathbf{B} & =-\mathbf{n} \cdot \mathbf{B}^{\text {inc }},
\end{aligned}
$$

where $\mathbf{n}$ denotes the unit normal to the scatterer.

Remark 1. While the tangential boundary conditions on $\mathbf{E}$ are sufficient to determine the electromagnetic field uniquely, we will see that it is convenient to make use of the condition on the normal component of $\mathbf{B}$ as well.

By analogy with the Lorenz-Mie-Debye potentials, let us seek a representation of the form

$$
\begin{aligned}
& \mathbf{E}=\nabla \times \nabla \times(\mathbf{r} v)-\frac{\partial}{\partial t} \nabla \times(\mathbf{r} u) \\
& \mathbf{B}=\nabla \times \nabla \times(\mathbf{r} u)+\frac{\partial}{\partial t} \nabla \times(\mathbf{r} v),
\end{aligned}
$$

where $\mathbf{r}=(x, y, z)$, while $u$ and $v$ are scalar functions of space-time. It is easy to check using standard vector identities that if $u$ and $v$ satisfy the scalar wave equation, then the representation (3) satisfies the Maxwell equations (1).

We now expand $u$ and $v$ in terms of spherical harmonics as

$$
\begin{aligned}
& u(r, \theta, \phi, t)=\sum_{n=0}^{\infty} \sum_{m=-n}^{n} u_{n m}(r, t) Y_{n m}(\theta, \phi), \\
& v(r, \theta, \phi, t)=\sum_{n=0}^{\infty} \sum_{m=-n}^{n} v_{n m}(r, t) Y_{n m}(\theta, \phi),
\end{aligned}
$$


where $(r, \theta, \phi)$ are the spherical coordinates of a point $\mathbf{r}=(x, y, z)$. Here,

$$
Y_{n}^{m}(\theta, \phi)=\sqrt{\frac{2 n+1}{4 \pi}} \sqrt{\frac{(n-|m|) !}{(n+|m|) !}} P_{n}^{|m|}(\cos \theta) e^{i m \phi},
$$

where $P_{n}(x)$ is the standard Legendre polynomial of degree $n$, and the associated Legendre functions $P_{n}^{m}$ are given by the Rodrigues' formula

$$
P_{n}^{m}(x)=(-1)^{m}\left(1-x^{2}\right)^{m / 2} \frac{d^{m}}{d x^{m}} P_{n}(x) .
$$

When clear from the context, we will often abuse notation and omit the arguments of the spherical harmonics, as well as the arguments of the functions $u_{n m}(r, t)$ and $v_{n m}(r, t)$. Applying the curl operator either once or twice to $\mathbf{r} u$, we obtain

$$
\begin{aligned}
\nabla \times(\mathbf{r} u) & =-\sum_{n=0}^{\infty} \sum_{m=-n}^{n} u_{n m} \boldsymbol{\Phi}_{n m} \\
\nabla \times \nabla \times(\mathbf{r} u) & =\sum_{n=0}^{\infty} \sum_{m=-n}^{n} \frac{n(n+1)}{r} u_{n m} \mathbf{Y}_{n m}+\left(\frac{\partial u_{n m}}{\partial r}+\frac{1}{r} u_{n m}\right) \boldsymbol{\Psi}_{n m} .
\end{aligned}
$$

The functions $\boldsymbol{\Psi}_{n m}, \boldsymbol{\Phi}_{n m}, \mathbf{Y}_{n m}$ are known as vector spherical harmonics, defined by

$$
\begin{aligned}
& \mathbf{Y}_{n m}(\theta, \phi)=Y_{n m}(\theta, \phi) \hat{\mathbf{r}}, \\
& \boldsymbol{\Psi}_{n m}(\theta, \phi)=r \nabla Y_{n m}(\theta, \phi), \\
& \mathbf{\Phi}_{n m}(\theta, \phi)=\mathbf{r} \times \nabla Y_{n m}(\theta, \phi),
\end{aligned}
$$

with $\hat{\mathbf{r}}$ the unit outward normal vector at $(\theta, \phi)$ (see, for example, [2]). In deriving (6), we have made use the following properties of vector spherical harmonics:

$$
\begin{aligned}
\nabla \times\left(f(r) \mathbf{Y}_{n m}\right) & =-\frac{1}{r} f \boldsymbol{\Phi}_{n m}, \\
\nabla \times\left(f(r) \boldsymbol{\Phi}_{n m}\right) & =-\frac{n(n+1)}{r} f \mathbf{Y}_{n m}-\left(\frac{d f}{d r}+\frac{1}{r} f\right) \boldsymbol{\Psi}_{n m} .
\end{aligned}
$$

From (6) and the analogous expressions for $v$, we have

$$
\begin{aligned}
& \mathbf{E}=\sum_{n=0}^{\infty} \sum_{m=-n}^{n} \frac{n(n+1)}{r} v_{n m} \mathbf{Y}_{n m}+\left(\frac{\partial v_{n m}}{\partial r}+\frac{1}{r} v_{n m}\right) \mathbf{\Psi}_{n m}+\frac{\partial u_{n m}}{\partial t} \boldsymbol{\Phi}_{n m}, \\
& \mathbf{B}=\sum_{n=0}^{\infty} \sum_{m=-n}^{n} \frac{n(n+1)}{r} u_{n m} \mathbf{Y}_{n m}+\left(\frac{\partial u_{n m}}{\partial r}+\frac{1}{r} u_{n m}\right) \mathbf{\Psi}_{n m}-\frac{\partial v_{n m}}{\partial t} \boldsymbol{\Phi}_{n m} .
\end{aligned}
$$


Because (a) vector spherical harmonics form an orthogonal basis for vector fields in $\mathbb{R}^{3}$ defined on the surface of a sphere, (b) $\hat{\mathbf{r}} \times \boldsymbol{\Psi}_{n m}=\boldsymbol{\Phi}_{n m}$ and (c) $\hat{\mathbf{r}} \times \boldsymbol{\Phi}_{n m}=-\boldsymbol{\Psi}_{n m}$, it is straightforward to verify that, on the unit sphere,

$$
\begin{aligned}
\mathbf{n} \cdot \mathbf{B} & =\sum_{n=0}^{\infty} \sum_{m=-n}^{n} n(n+1) u_{n m}(1, t) Y_{n m}, \\
\mathbf{n} \times \mathbf{E} & =\sum_{n=0}^{\infty} \sum_{m=-n}^{n}\left(\frac{\partial v_{n m}}{\partial r}(1, t)+v_{n m}(1, t)\right) \boldsymbol{\Phi}_{n m}-\frac{\partial u_{n m}}{\partial t}(1, t) \boldsymbol{\Psi}_{n m} .
\end{aligned}
$$

Thus, $u_{n m}(1, t)$ is determined entirely by the projection of the normal component of the magnetic field onto the corresponding spherical harmonic. As we shall see in the next section, this Dirichlet boundary data is sufficient to determine $u_{n m}(r, t)$ for all $r>1$ and all time $t>0$. Likewise, the projection of $\mathbf{n} \times \mathbf{E}$ onto $\boldsymbol{\Phi}_{n m}$ determines $\frac{\partial v_{n m}}{\partial r}(1, t)+v_{n m}(1, t)$, and this Robin boundary data is sufficient to determine $v_{n m}(r, t)$ for all $r>1$ and all time $t>0$.

\section{Evaluation of the radial functions $u_{n m}(r, t)$ and $v_{n m}(r, t)$}

Following the approach of [6], we briefly describe the computation of $u_{n m}(r, t)$ and $v_{n m}(r, t)$.

Theorem 1. [6] Let $\alpha_{n, j}$ for $j=1, \ldots, n$ denote the roots of the modified spherical Hankel function $k_{n}$ [14] (which are simple and lie in the open left half of the complex plane). Further, let

$$
a_{n, j}(r)=r e^{\alpha_{n, j}(r-1)} \frac{k_{n}\left(\alpha_{n, j} r\right)}{k_{n}^{\prime}\left(\alpha_{n, j}\right)} .
$$

Then

$$
u_{n m}(r, t)=\frac{1}{r}\left(f_{n m}(t-r+1)+\sum_{j=1}^{n} a_{n, j}(r) \int_{0}^{t-r+1} e^{\alpha_{n, j}(t-r+1-\tau)} f_{n m}(\tau) d \tau\right),
$$

where $f_{n m}(t)=u_{n m}(1, t)$.

The main result of [6] was the derivation of the following stable recurrence formula for $u_{n m}(r, t)$ :

$$
\begin{aligned}
& \phi_{0}(r, t)=f_{n m}(t), \\
& \phi_{j}(r, t)=\phi_{j-1}(r, t)+\left(1-\frac{1}{r}\right) \alpha_{n, j} \int_{0}^{t} e^{\alpha_{n, j}(t-\tau)} \phi_{j-1}(r, \tau) d \tau,
\end{aligned}
$$


for $j=1, \ldots, n$, with

$$
u_{n m}(r, t)=\frac{1}{r} \phi_{n}(r, t-r+1) .
$$

For stability, the roots $\alpha_{n, j}$ of the modified spherical Hankel function $k_{n}(z)$ should be arranged in ascending order according to their real parts. That is, $\alpha_{n, 1}$ should be the root with the largest negative real part and $\alpha_{n, n}$ should be the root with the smallest negative real part. axis.

A similar result holds for $v_{n m}(r, t)$. If we let $g_{n m}(t)=\frac{\partial v_{n m}}{\partial r}(1, t)+v_{n m}(1, t)$, then $v_{n m}(r, t)$ can be computed using the following recurrence [6]:

$$
\begin{aligned}
& \psi_{0}(r, t)=\int_{0}^{t} e^{\beta_{n, 0}(t-\tau)} g_{n m}(\tau) d \tau \\
& \psi_{j}(r, t)=\psi_{j-1}(r, t)+\left(\beta_{n, j}-\frac{1}{r} \alpha_{n, j}\right) \int_{0}^{t} e^{\beta_{n, j}(t-\tau)} \psi_{j-1}(r, \tau) d \tau,
\end{aligned}
$$

for $j=1, \cdots, n$, with

$$
v_{n m}(r, t)=-\frac{1}{r} \psi_{n}(r, t-r+1) .
$$

Here, $\beta_{0,0}=0$ and $\beta_{n, j}$ are the simple roots of the function $D_{n}(z)=z k_{n}^{\prime}(z)+$ $k_{n}(z)$ for $j=0, \ldots, n$ when $n>0$. They must also be arranged in ascending order according to their real parts.

Remark 2. An alternative algorithm for evaluating the radial functions arising in the solution of the scalar wave equation, based on a compressed sumof-poles representation, has been proposed in [8]. With modification it could also be used to compute the Debye potentials.

\section{Solution of the full time-dependent Maxwell equations}

We may now summarize our principal result.

Theorem 2. Suppose that $\mathbf{E}$ and $\mathbf{B}$ satisfy the time-dependent Maxwell equation outside the perfectly conducting unit sphere

$$
\begin{aligned}
\nabla \times \mathbf{E} & =-\frac{\partial \mathbf{B}}{\partial t}, & \nabla \times \mathbf{B} & =\frac{\partial \mathbf{E}}{\partial t}, \\
\nabla \cdot \mathbf{E} & =0, & \nabla \cdot \mathbf{B} & =0,
\end{aligned}
$$


subject to the boundary conditions

$$
\begin{aligned}
\mathbf{n} \cdot \mathbf{B} & =\sum_{n=0}^{\infty} \sum_{m=-n}^{n} n(n+1) f_{n m} Y_{n m}, \\
\mathbf{n} \times \mathbf{E} & =\sum_{n=0}^{\infty} \sum_{m=-n}^{n} g_{n m} \boldsymbol{\Phi}_{n m}-\frac{\partial f_{n m}}{\partial t} \boldsymbol{\Psi}_{n m}
\end{aligned}
$$

with zero initial data at time $t=0$. Suppose further that $\mathbf{E}$ and $\mathbf{B}$ are represented via Debye-like potentials $u, v$ :

$$
\begin{aligned}
& \mathbf{E}=\nabla \times \nabla \times(\mathbf{r} v)-\frac{\partial}{\partial t} \nabla \times(\mathbf{r} u) \\
& \mathbf{B}=\nabla \times \nabla \times(\mathbf{r} u)+\frac{\partial}{\partial t} \nabla \times(\mathbf{r} v) .
\end{aligned}
$$

Then, (15) provides a solution to the scattering problem (13), (14) if $u$ and $v$ are given by

$$
\begin{aligned}
& u(r, \theta, \phi, t)=\sum_{n=1}^{\infty} \sum_{m=-n}^{n} u_{n m}(r, t) Y_{n m}(\theta, \phi), \\
& v(r, \theta, \phi, t)=\sum_{n=1}^{\infty} \sum_{m=-n}^{n} v_{n m}(r, t) Y_{n m}(\theta, \phi),
\end{aligned}
$$

where $u_{n m}, v_{n m}$ are computed via $(9,10)$, and (11,12), respectively.

In order to compute the electric and magnetic fields from (7), we will also need to evaluate $\frac{\partial u_{n m}}{\partial t}, \frac{\partial u_{n m}}{\partial r}+\frac{1}{r} u_{n m}, \frac{\partial v_{n m}}{\partial t}$, and $\frac{\partial v_{n m}}{\partial r}+\frac{1}{r} v_{n m}$. From above, we have

$$
\frac{\partial u_{n m}}{\partial t}=\frac{1}{r} \frac{\partial \phi_{n}(r, t-r+1)}{\partial t} .
$$

For the computation of the partial derivative, it is straightforward to derive the formula

$$
\begin{aligned}
\frac{\partial \phi_{n}(r, t)}{\partial t} & =f_{n m}^{\prime}(t)-\frac{\alpha_{n, 1}}{r} f_{n m}(t) \\
& +\sum_{j=1}^{n-1}\left(\alpha_{n, j}-\alpha_{n, j+1} / r\right) \phi_{j}(r, t)+\alpha_{n, n} \phi_{n}(r, t) .
\end{aligned}
$$


In eq. (2.16) of [1], it is shown that each mode $u_{n m}$ is outgoing and satisfies the one way advection equation:

$$
\frac{\partial u_{n m}}{\partial r}(r, t)+\frac{\partial u_{n m}}{\partial t}(r, t)+\frac{1}{r} u_{n m}(r, t)=\int_{0}^{t} \omega_{n}(r, t-\tau) u_{n m}(r, \tau) d \tau,
$$

where $\omega_{n}$ is the convolution kernel for the exact nonreflecting boundary condition for the wave equation in three dimensions. That kernel is defined by the formula

$$
\omega_{n}(r, t)=\frac{1}{r^{2}} \sum_{j=1}^{n} \alpha_{n, j} e^{\frac{\alpha_{n, j}}{r} t} .
$$

Thus

$$
\begin{aligned}
\frac{\partial u_{n m}}{\partial r}(r, t)+\frac{1}{r} u_{n m}(r, t) & =-\frac{\partial u_{n m}}{\partial t}(r, t) \\
& +\frac{1}{r^{2}} \sum_{j=1}^{n} \alpha_{n, j} \int_{0}^{t} e^{\alpha_{n, j}(t-\tau) / r} u_{n m}(r, \tau) d \tau
\end{aligned}
$$

For the $v_{n m}$ terms, note first that

$$
\frac{\partial v_{n m}}{\partial t}=-\frac{1}{r} \frac{\partial \psi_{n}(r, t-r+1)}{\partial t} .
$$

The computation of the partial derivative is again straightforward to derive:

$$
\frac{\partial \psi_{n}(r, t)}{\partial t}=g_{n m}(t)+\sum_{j=1}^{n}\left(\beta_{n, j-1}-\alpha_{n, j} / r\right) \psi_{j-1}(r, t)+\beta_{n, n} \psi_{n}(r, t) .
$$

Finally, we observe that $v_{n m}$, like $u_{n m}$, satisfies the one way advection equation (19). Thus,

$$
\begin{aligned}
\frac{\partial v_{n m}}{\partial r}(r, t)+\frac{1}{r} v_{n m}(r, t) & =-\frac{\partial v_{n m}}{\partial t}(r, t) \\
& +\frac{1}{r^{2}} \sum_{j=1}^{n} \alpha_{n, j} \int_{0}^{t} e^{\alpha_{n, j}(t-\tau) / r} v_{n m}(r, \tau) d \tau .
\end{aligned}
$$




\section{Numerical examples}

A high order accurate solver for the time-domain Maxwell equations has been written in Fortran, based on the method described above. We use standard recurrence relations ([14], 14.10) to evaluate spherical harmonics $Y_{n}^{m}(\theta, \phi)$. A method for the computation of the zeros of $k_{n}(z)$ and $D_{n}(z)$ is described in some detail in [6]. Finally, we achieve $p$ th order accuracy in time by approximating the functions $f_{n m}(t), \phi_{j}(r, t), g_{n m}(t), \psi_{j}(r, t), u_{n m}(r, t)$, and $v_{n m}(r, t)$ by piecewise $p$ th order polynomials in time and compute all integrals exactly for this approximation. This approach is often referred to as product integration and we omit detailed formulas, referring the reader again to [6].

Example 1: Convergence AND STABility STUdy.

To test the convergence and stability of our algorithm, we consider the fields generated by a dipole $\mathbf{p}_{0}$ located at $\mathbf{y}$ with time signal $f(t)$. The analytical expressions for electric and magnetic fields are given by the formulas [12]

$$
\begin{aligned}
\mathbf{E}(\mathbf{x}, t) & =\left[\left(\mathbf{p}_{0} \cdot \hat{\mathbf{r}}\right) \hat{\mathbf{r}}-\mathbf{p}_{0}\right] \frac{f^{\prime \prime}(t-r)}{r}+\left[3\left(\mathbf{p}_{0} \cdot \hat{\mathbf{r}}\right) \hat{\mathbf{r}}-\mathbf{p}_{0}\right]\left(\frac{f^{\prime}(t-r)}{r^{2}}+\frac{f(t-r)}{r^{3}}\right), \\
\mathbf{H}(\mathbf{x}, t) & =\left[\mathbf{p}_{0} \times \hat{\mathbf{r}}\right]\left(\frac{f^{\prime \prime}(t-r)}{r}+\frac{f^{\prime}(t-r)}{r^{2}}\right),
\end{aligned}
$$

where $r=|\mathbf{x}-\mathbf{y}|, \hat{\mathbf{r}}=\frac{\mathbf{x}-\mathbf{y}}{r}$, and $f^{\prime}, f^{\prime \prime}$ are derivatives with respect to time. Here the exact solution is generated by two dipoles located at $\mathbf{y}=$ $(0.2,-0.5,0.6)$ with $\mathbf{p}_{0}=(1.8,-0.3,0.7)$ and $\mathbf{y}=(-0.4,-0.5,0.7)$ with $\mathbf{p}_{0}=(0.65,-0.5,1.5)$, respectively. The time signal is of the form $f(t)=$ $e^{-\left(t-t_{0}\right)^{2} / a} \cos (k t)$. The first dipole has $t_{0}=1.6, a=0.05 d 0$, and $k=100$, and the second dipole has $t_{0}=3.2, a=0.28 d 0$, and $k=80$. The numerical solution is evaluated on a sphere of radius $r=100$ at $T=103$.

Tables 1-2 show the relative $L^{2}$ error of the numerical solution for varying values of $N$, the order of the spherical expansion and the total number of time steps. Note that the solution is oscillatory in both space and time, so that finite difference or finite element methods would have difficulty computing the solution in the far field with high precision because of numerical dispersion errors. In particular, there are approximately 1590 wavelengths between the scatterer and the evaluation sphere. In Table 1, the order of temporal 
convolution is $p=20$ and we break the shifted time interval $[0, t-r+1]=$ $[0,4]$ into 100 equispaced subintervals (yielding a total of 2000 discretization points in time). In Table 2, we fix the order of the spherical harmonic expansion at 125 , to test the temporal convergence rate.

\begin{tabular}{|c|c|c|c|c|c|c|}
\hline$N_{S}$ & 102400 & 129600 & 160000 & 193600 & 230400 & 270400 \\
\hline$N$ & 80 & 90 & 100 & 110 & 120 & 130 \\
\hline$E$ & $0.11 \mathrm{E}+00$ & $0.11 \mathrm{E}-02$ & $0.20 \mathrm{E}-05$ & $0.11 \mathrm{E}-08$ & $0.14 \mathrm{E}-11$ & $0.14 \mathrm{E}-11$ \\
\hline
\end{tabular}

Table 1: Relative $L^{2}$ error of the numerical solution to the Maxwell equations as a function of the spherical harmonic order $N$ and the number of discretization points $N_{S}$ on the unit sphere. Since the discretization error is usually greater than the truncation error, we set $N_{\theta}=N_{\phi}=4 N$, so that $N_{S}=16 N^{2}$. The total number of discretization points in time is $N_{T}=2000$ and the order of temporal integration is $p=20$.

\begin{tabular}{|c|c|c|c|c|c|c|}
\hline$N_{T}$ & 300 & 400 & 800 & 1200 & 1600 & 2000 \\
\hline$E$ & $0.11 \mathrm{E}-01$ & $0.65 \mathrm{E}-04$ & $0.18 \mathrm{E}-09$ & $0.12 \mathrm{E}-11$ & $0.25 \mathrm{E}-11$ & $0.14 \mathrm{E}-11$ \\
\hline
\end{tabular}

Table 2: Relative $L^{2}$ error of the numerical solution to the Maxwell equations as a function of the number of points in the time discretization. The order of the spherical harmonic expansion is fixed at 125 and the total of number of discretization points on the unit sphere is set to $N_{S}=250000$. The order of integration for temporal convolution is $p=20$.

Figure 1 shows timing results for a single core on a $2.1 \mathrm{GHz}$ laptop computer. The left panel shows that the time per step is in a good agreement with the theoretical result $O\left(N_{S}^{3 / 2}\right)$ with $N_{S}$ the total number of discretization points in space; and the right panel shows that the total simulation time is linear with respect to the total number of time steps $N_{T}$. Thus, the complexity of the algorithm is $O\left(N_{S}^{3 / 2} N_{T}\right)$.

EXAmple 2: ScAtTERING OF A PUlSE.

In this example, we consider the scattering of a pulse by the perfectly conducting unit sphere with boundary data generated by the following incident wave:

$$
\begin{aligned}
& \mathbf{A}=\hat{j} A(z, t)=\hat{j} e^{i k(z-t)} e^{-\left(z-t+t_{c}\right)^{2} / a} \\
& \mathbf{E}=-\frac{\partial \mathbf{A}}{\partial t}=-\hat{j} \frac{\partial A(z, t)}{\partial t}=\hat{j} \frac{\partial A(z, t)}{\partial z} \\
& \mathbf{B}=\nabla \times \mathbf{A}=\hat{i} \frac{\partial A(z, t)}{\partial z}
\end{aligned}
$$



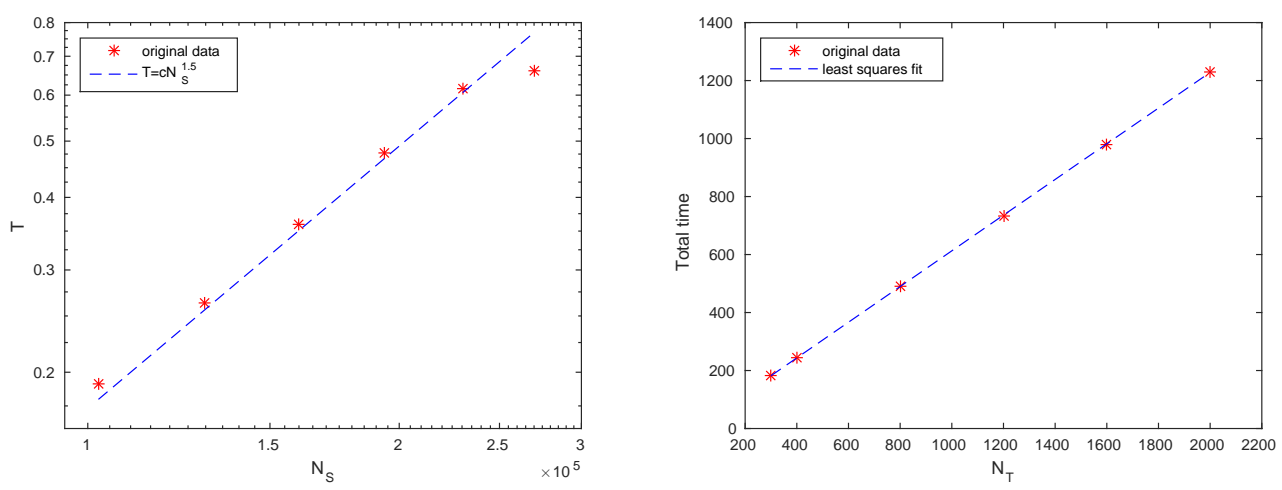

Figure 1: Timing result for Example 1. The left panel shows the time per step $T$ versus the total number of discretization point $N_{S}$ on the unit sphere; the $*$ points are the simulation results and the dashed line is the theoretical result $O\left(N_{S}^{3 / 2}\right)$. The right one shows the total simulation time versus the total number of time steps $N_{T}$; the $*$ points are the simulation results and the dashed line is the least squares line fitting of the data.

where $\mathbf{A}$ is the vector potential, and $\hat{i}, \hat{j}$ are the unit vectors along the $x, y$ directions, respectively. We choose $k=100, t_{c}=1.6, a=0.05$ and compute the (axisymmetric) pattern of the scattered field as a function of angle at $R=1.1$ and at $R=1000$ at $T=t_{c}+R-1$. In the latter case, the solution is being computed about 16,000 wavelengths away from the scatterer (Fig. 2 ). The order of the spherical harmonic expansion is 150; the total number of discretization points in time is $N_{T}=2000$ and the order of temporal integration is $p=20$.

\section{Conclusions}

We have extended the Lorenz-Mie-Debye representation to the timedomain and, as a result, derived an explicit solution to the problem of transient electromagnetic scattering from a perfectly conducting sphere. Our representation leads to a stable and high-order numerical scheme, making use of the algorithm of [6] for the scalar (time-domain) wave equation. It is worth noting that previous approaches to the scalar problem $[15,17,20]$ were generally unsuitable for computation when considering high order spherical harmonics, due to numerical instabilities (discussed in more detail in $[6,8]$ ). It is, perhaps, due to these difficulties that separation of variables methods in the literature have historically been limited to the frequency domain. It 

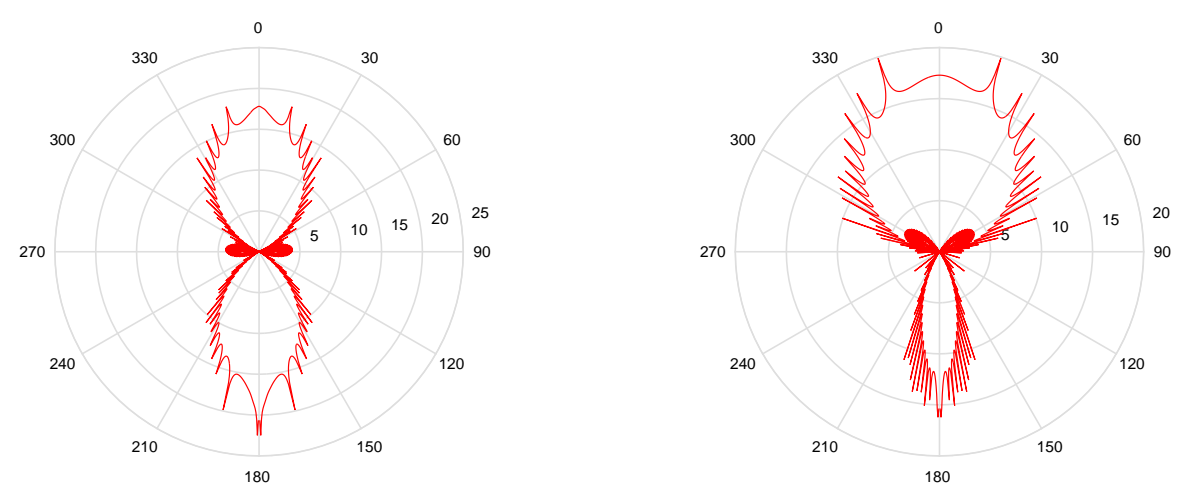

Figure 2: The scattered electric field $u$ due to an incident pulse traveling in the $z$-direction. We plot the scaled quantity $\log (R|\mathbf{E}(R, \theta, T)|)$ as a function of polar angle in the near field $R=1.1$ (left) and in the far field $R=1000$ (right).

is also worth noting, however, that alternative stable and high order methods for time-domain scattering from a sphere have recently been constructed using integral equation methods. The paper [9] considers the scalar wave equation and the paper [10] considers the full Maxwell equations. While not explicit (in the sense of writing down the solution operator as a function acting on the data), the authors make use of a spherical harmonic decomposition of an unknown time-dependent source or current distribution on the sphere and, for each mode, derive a Volterra integral equation to solve for those unknowns. We suspect that both our scheme and this integral equation approach will provide insight when seeking to develop high order methods for more general geometries.

Finally, we note that standard perturbation theory can be combined with the method developed in this paper to solve the time-dependent Maxwell equations when the scatterer is a mildly deformed sphere. In general, however, time-domain integral equations will be necessary (see, for example, $[3,16,19])$.

\section{References}

[1] B. Alpert, L. Greengard, and T. Hagstrom, Rapid evaluation of nonreflecting boundary kernels for time-domain wave propagation, SIAM J. Numer. Anal. 37 (2000), 1138-1164.

[2] E. Carrascal, G. A. Estevez, P. Lee, and V. Lorenzo, Vector spheri- 
cal harmonics and their application to classical electrodynamics, Eur. J. PHys. 12 (1991), 184-191.

[3] M. Costabel, Time-dependent problems with the boundary integral equation method, in E. Stein, R. de Borst, T. J. R. Hughes (eds), Encyclopedia of Computational Mechanics, (2004), 1-28.

[4] P. Debye, Der Lichtdruck auf Kugeln von beliebigen Material, Ann. Phys. (Leipzig) 30 (1909), 57-136.

[5] C. Epstein and L. Greengard, Debye Sources and the Numerical Solution of the Time Harmonic Maxwell Equations, Comm. Pure Appl. Math. 63 (2010), 413-463.

[6] L. Greengard, T. Hagstrom, and S. Jiang, The solution of the scalar wave equation in the exterior of a sphere, J. Comput. Phys. 274 (2014), 191-207.

[7] J. D. Jackson, Classical Electrodynamics, John Wiley \& Sons, New York, 1975 .

[8] S. Field and S. Lau, Fast evaluation of far-field signals for time-domain wave propagation, preprint, arXiv:1409.5893 [math.NA].

[9] J. Li, D. Dault, and B. Shanker, A quasianalytical time domain solution for scattering from a homogeneous sphere, J. Acoust. Soc. Am. 135 (2014), 1676-1685.

[10] J. Li and B. Shanker, Time-dependent Debye-Mie series solutions for electromagnetic scattering, preprint, arXiv:1412.8171 [math.NA].

[11] L. V. Lorenz, Lysvevxgelsen $i$ og uden for en af plane lysbolger belyst kugle, K. Dan. Vidensk. Selsk. Forh. 6 (1890), 1-62.

[12] K. T. McDonald, The fields of a pulsed, small dipole antenna, Lecture Notes, Joseph Henry Laboratories, Princeton University.

[13] G. Mie, Beiträge zur Optik trüber Medien, speziell kolloidaler Metallösungen, Ann. Phys. (Leipzig) 25 (1908), no. 3, 377-445. 
[14] F. W. J. Olver, D. W. Lozier, R. F. Boisvert, and C. W. Clark, editors. NIST Handbook of Mathematical Functions, Cambridge University Press, New York, NY, 2010.

[15] S. Sauter and A. Veit, A Galerkin method for retarded boundary integral equations with smooth and compactly supported temporal basis functions Numer. Math. (2013) 123, 145-176.

[16] B. Shanker, A. Ergin, and E. Michielssen, Fast analysis of transient electromagnetic scattering phenomena using the multilevel plane wave time domain algorithm, IEEE Transactions on Antennas and Propagation 51 (2003), 628-641.

[17] T. Tokita, Exponential decay of solutions for the wave equation in the exterior domain with spherical boundary, J. Math. Kyoto Univ. 12-2 (1972), 413-430.

[18] M. Tygert, Fast algorithms for spherical harmonic expansions, III, J. Comput. Phys. 229 (2010), no. 18, 6181-6192.

[19] F. Valdés, M. Ghaffari-Miab, F. P. Andriulli, K. Cools, and E. Michielssen, High-order Calderón preconditioned time domain integral equation solvers, IEEE Transactions on Antennas and Propagation 61 (2013), 2570-2588.

[20] C. H. Wilcox, The initial-boundary value problem for the wave equation in an exterior domain with spherical boundary, Notices Amer. Math. Soc. 6 (1959), 869-870. 